\title{
La importancia del capital humano y su base legal dentro de las organizaciones
}

\section{Resumen}

El objetivo de la investigación es analizar la importancia del capital humano en las organizaciones, tomando en consideración la base legal desde el punto de vista laboral, y la relevancia del conocimiento relacionados con los deberes y derechos que, en muchos casos, se encuentra estipulados en las leyes, decretos constitucionales y, que rigen de alguna manera, el direccionamiento hacia el trabajo y la vida digna del capital humano. Es una investigación de tipo cualitativa-interpretativa basado en la hermenéutica, sobre los ejes temáticos que intervienen en el estudio. Mediante el método interpretativo, se pudo evidenciar, la subjetividad del estudio, además de rescatar la importancia de las acciones y del comportamiento de los sujetos investigados. Los resultados del estudio, expresan que el análisis y el conocimiento de las leyes, decretos y gacetas relacionadas con la legalidad laboral, evitan la explotación del hombre por el hombre. Y finalmente, se resalta la complejidad tanto del 
capital humano como su legalidad en el entorno laboral, para destacar la prosecución de objetivos gerenciales, con la intencionalidad de realzar la competitividad, la innovación y sobre todo la responsabilidad social de los trabajadores.

Palabras clave: Capital humano, complejidad, legislación laboral, responsabilidad social.

\begin{abstract}
The objective of the research is to know the importance of human capital in organizations, taking into consideration the legal basis from the labor point of view, and its relevance to the knowledge of their duties and rights that, in many cases, are stipulated in the laws, constitutional decrees and, which govern in some way, the direction towards work and the dignified life of human capital. It is a qualitative-interpretative research based on hermeneutics, on the thematic axes involved in the study. Among the purposes of the research is to develop the importance and meaning that human capital has towards organizational growth. Unveil laws, decrees and gazettes related to labor legality, with the intention that human capital knows its rights before the employer; in order to avoid the exploitation of man towards man. And finally, highlight the complexity of both human capital and its legality in the workplace, as a panacea towards the pursuit of management objectives, with the sole intention of enhancing competitiveness, innovation and especially social responsibility, to dignify the work of the fundamental matter in organizations as it is, human capital.
\end{abstract}

Keywodrs: Human capital, complexity, labor legislation, social responsibility. 


\section{Introducción}

El capital humano es considerado como referencia que afecta parte de los ecosistemas, recursos naturales y su propio hábitat (Etkin, 2005). La complejidad en el capital humano, obliga a las organizaciones a preparar estrategias gerenciales en todos sus ámbitos operativos, desde el punto de vista de la organización, lo cual debería ir en concordancia con los objetivos de desarrollo sustentables establecidos por la gerencia.

La efectividad de las empresas, será posible, según manejen de manera inteligente su capital humano, adecuando los mismos a todos sus procesos y, haciéndolos partícipe en los proyectos organizacionales. En este sentido, la inversión del futuro como primera prioridad, deberá ser precisamente la capacitación del capital humano, donde los beneficios empresariales retornarán con mayor rapidez y, por ende, el factor de competitividad se afianzará en sus procesos.

Cuando hablamos de capital humano en las organizaciones, el mismo debe ser interpretado como factor clave integrador hacia el cambio, con el fin de explorar las competencias, el comportamiento, habilidades y destrezas que se debe tener para enfrentar las diferentes situaciones operativas de las empresas. En este sentido Vargas (2002), señala que las experiencias exitosas del capital humano suelen residir en la habilidad de la organización para establecer un marco de capacidades que refleje su filosofía, valores y objetivos estratégicos.

Desde el punto de vista legal laboral, el capital humano se rige por el conjunto de normas jurídicas que respaldan sus deberes y derechos con el patrón y viceversa. Este conjunto de normas, representan un vínculo del derecho jurídico, por la dependencia de hacer cumplir las leyes y las diferentes regulaciones por ambas partes. Lo que quiere decir que a pesar de existir una figura dependiente, debe existir una subordinación sujeta legalmente, que permita establecer de manera coherente y armoniosa, una relación de trabajo digna.

La legalidad del capital humano en las organizaciones, surge por la necesidad de realzar las luchas hacia la igualdad y el crecimiento personal de los trabajadores, con la intención de que le sean reconocidos sus derechos y de obtener mejores condiciones laborales. La idea es regular su situación frente a sus obligaciones frente al patrón y terminar finalmente, con la explotación del hombre por el hombre.

El método empleado para llevar a cabo esta investigación es el interpretativo, el cual permite teorizar sobre la práctica del sujeto, grupo o sociedad. Al mismo tiempo, intenta comprender las conductas desde el punto de vista de los actores, desde su percepción (Dulzaides y Molina, 2004).

Por medio del método, podemos profundizar en los diferentes motivos, significaciones y sentido a las conductas o comportamientos desde una mirada del contexto dentro del cual se sitúa. Finalmente, enmarcamos las conclusiones sobre el conjunto de ideas sobre los ejes temáticos, que permiten analizar el marco teórico-conceptual, fundamentado en la interpretación del objeto de estudio.

Los resultados de la investigación vienen dados, no sólo por la importancia que representa el 
capital humano para las organizaciones, sino también por la necesidad de que ese capital humano conozca mediante las diferentes leyes, decretos y gacetas jurídicas, sus deberes y derechos ante el patrón, con la finalidad de enfrentar de manera justa, el trabajo digno que permita el crecimiento personal y, por ende, del grupo familiar. Es importante dar a conocer los diferentes procesos laborales existentes, según el país o región donde se desenvuelve, para conocer sus derechos y deberes; para evitar la explotación y el engaño por parte de algunos patrones inescrupulosos, que se mantienen al margen de la ley.

\section{La importancia del capital humano para las organizaciones}

Las organizaciones por lo general, procuran la estabilidad económica, por medio de herramientas gerenciales que les permiten estructurar de manera efectiva, una serie de normas y políticas que acompañen la visión y misión del negocio (Alfonso, 2019). El capital humano forma parte de esas políticas intrapersonales y profesionales, que deben ser tomadas en cuenta para apoyar de manera constante las expectativas comerciales, por lo tanto, la relación patrono/trabajador, se convierte en un elemento central para el punto de equilibrio organizacional.

Las organizaciones deben apuntar hacia el logro de la estabilidad y regularidad de sus procesos, de esta manera, el entorno general de todos sus componentes internos y externos (administrativos, operacionales, financieros y recursos humanos) podrán adecuarse y adaptarse a los diferentes cambios de entornos que enfrentan (Torres 2018). Cuando se presentan cambios significativos organizacionales y, sobre todo cuando no se está preparado para enfrentarlos, inmediatamente se hace presente la incertidumbre; lo cual atenta directamente contra la estabilidad de quienes integran a la empresa.

La incertidumbre es capaz de reducir la planificación desde el punto de vista estratégico y previsivo, es decir, a mayor incertidumbre organizacional mayor pudieran ser sus costos operacionales que influyen directamente en la rentabilidad del negocio y, de manera colateral, en la estabilidad del capital humano como factor integrador de la responsabilidad social empresarial (Kin, 2017).

Desde el punto de vista de la responsabilidad social, el capital humano forma parte importante para contrarrestar esa incertidumbre que sólo trae como consecuencia la contracción en el crecimiento de la organización (Godin, 2015). El capital humano debe volverse parte de la revisión constante de las políticas, estrategias y prácticas gerenciales, para contrarrestar esta situación y así reconvertirlas en acciones productivas y de crecimiento (Sull, Homkes y Sull, 2015).

Por lo tanto, una organización sin su capital humano puede significar ir en contra de la corriente, prácticamente podría dejar de existir (Blanco, 2017). La cantidad de personas existente en una empresa, dependerá de las necesidades de la misma. Los conocimientos, actitudes y compromiso del capital humano hacia la organización, quedarán sujetos para decidir en muchos casos, el éxito y la prosecución de los objetivos gerenciales (Tushman, Smith y Binns, 2011).

Si todo marcha de manera positiva y correcta en tiempos normales, los resultados en tiempos volátiles e inciertos serán acordes a las exigencias del momento. Por ello, es fundamental 
aproximarse al capital humano con disposiciones claras y precisas, para poder formar ese trabajo en equipo fundamental para el crecimiento organizacional (López y Grandío, 2005).

Generalmente, las organizaciones tratan de mantener la relación laboral de manera estable y consistente, dándole importancia relativa a su personal y viéndolos como la materia prima principal para obtener un punto de equilibrio intelectual (Barroso, 2011). Parte de esa relación, se logra con políticas salariales, así como de incentivos que van acorde a las responsabilidades y a la economía del país. De esta forma el trabajador se concentra de forma objetiva en su trabajo y da lo mejor de sí para perdurar en su puesto y lograr una independencia económica que lo proyecte hasta su retiro.

Existen algunas creencias organizacionales, que sostienen que explorar nuevas oportunidades en el proceso productivo, es algo innecesario y que no aporta nada positivo al crecimiento organizacional (Francés, 2008). Empero, hay organizaciones que exploran más allá de sus expectativas, y buscan en su capital humano, ese plus extra que les permita explorar en la innovación, la competitividad y el emprendimiento, demostrando que si se pueden emplear sistemas que funcionen como eje proveedor de personas con actitud emprendedora para los distintos departamentos.

La idea es, la resolución de problemas y conflictos, así como la mejora en sus procesos con el fin de rediseñar y replantear escenarios de cambio, que solidifiquen a la organización. Todo este proceso tiene como norte, conocer las dificultades, incomodidades y problemas que el capital humano padece en su sitito de trabajo, trayendo como consecuencia ruido y des-productividad que atenta con los objetivos planteados.

Debe existir un binomio bien armonizado, que permita evolucionar en todos los aspectos de la organización y, el capital humano, es importante en dicho proceso (Penfold y Vainrub, 2012). Entre las variables que distorsionan este proceso de eje emprendedor, se encuentran los cambios técnicos, culturales y comunicacionales, los cuales deben ser manejados de manera inteligente por los líderes divisionales, para convertirlos en fortalezas y, que al mismo tiempo, sirvan como ente integrador organizacional.

Finalmente, la importancia que reviste el capital humano para las organizaciones, va desde el aporte intelectual hasta su aporte productivo. Y para eso pueden surgir y deben ocurrir una serie de elementos integrados que ambas partes patrón y trabajador deben poner en práctica para poder solventar situaciones que coadyuven a la resolución de conflictos y adversidades que se presenten en la organización. La empresa para mejorar sus procesos tantos internos como externos, también debe invertir y creer en su capital humano, por lo tanto, debe hacer inversiones de crecimiento en cursos y especializaciones que aporten de manera positiva los procesos (Etkin y Schvarstein, 2000).

Para concretar proyectos prospectivos, es necesario mucha conversación y comunicación con todos los integrantes de la empresa (Firth, 2000). La mejora continua fortalece al eje emprendedor, porque posibilita las cosas que son realmente importantes. Gerenciar personas no es tarea fácil (Drucker, 2000). Exige, entre otras cosas, enfrentar la inercia, seguir haciendo 
lo que siempre se ha hecho. Significa luchar contra lo obvio que condena a no ver más allá de creencias que condenan a seguir repitiendo, sin cuestionamiento, lo que ya no sirve porque las circunstancias han cambiado de manera radical.

\section{El capital humano y su relevancia legal organizacional en Ecuador}

El capital humano desde el punto de vista legal, se encuentra amparado por una serie de leyes sociales e instituciones gubernamentales, donde la intención es amparar al trabajador, para que no se le vulneren sus derechos (López, 2015). Estas leyes sociales pueden variar según la perspectiva o puntos de vista existentes en los diferentes países donde se ejecuten e implante.

Sin embargo con este punto, García (2015) expresa que existen muchas similitudes o congruencias que pretenden proteger al trabajador tomando en cuenta su salario, sus beneficios sociales (prestaciones sociales, vacaciones, utilidades, bonos, horarios, horas extras, liquidaciones, etcétera) y crecimiento intelectual, en función de desarrollarse dentro de las organizaciones.

Desde el punto de vista legal, las empresas se encuentran obligadas a respetar ciertos derechos laborales al trabajador, para evitar incurrir en multas y suspensiones (Buen, 2015). Expresa el autor que la legalidad laboral comienza donde acaban los derechos, por lo tanto no sólo las organizaciones deben velar por el cumplimiento de sus metas gerenciales, sino que también, deben cumplir con una serie de beneficios al trabajador y, de esta manera, compensar de forma justa los objetivos entre ambas partes.

Al respecto, Bazán, (1997) denota un principio de legalidad en el ámbito jurídico que se fundamenta dentro del derecho público, el cual tiene por objeto sustentar de manera consistente y legal, todas aquellas normas jurídicas para lograr el ordenamiento de la sociedad en general, inclusive determinando órganos competentes jurisdiccionales encargados de hacer velar las mismas en pro de un sistema legal sano y competente.

Esta razón legal comentada anteriormente, es la que le permite al Estado, que se cumplan en este caso, todas las legalidades existentes en el entorno laboral, para reguardar la seguridad jurídica del capital humano que labora en las organizaciones. Por lo tanto, es importante partir de este principio de legalidad ya que se comporta como parámetro legislador para el cumplimiento de las leyes dentro de Estado de derecho, donde se puede ejercer de manera contundente los fundamentos legales y conocer al mismo tiempo, los límite de todos aquellos que deben hacerlas cumplir (Palomo, 2008).

Por ejemplo en Ecuador, cuando investigamos respecto a la legalidad del derecho laboral en el capital humano organizacional, lo primero que se resalta es lo que se encuentra estipulado en la Constitución de la República (2008) en el artículo Nro. 66 numeral 2 el cual contempla que el reconocimiento hacia las personas en cuanto a: El derecho a una vida digna, que asegure la salud, alimentación, nutrición, agua potable, vivienda, saneamiento ambiental, educación, trabajo, empleo, descanso y ocio, cultura física, vestido, seguridad social y otros servicios sociales necesarios. 
Así como también en el artículo Nro. 33 de la citada Constitución estipula que:

El trabajo es un derecho y un deber social, y un derecho económico, fuente de realización personal y base de la economía. El Estado garantizará a las personas trabajadoras el pleno respeto a su dignidad, una vida decorosa, remuneraciones y retribuciones justas y el desempeño de un trabajo saludable y libremente escogido o aceptado.

Lo que se pretende como Estado, es resguardar y asegurar de manera legal, todo lo relacionado a la seguridad social del capital humano en las empresas, con el fin de hacer cumplir los derechos constitucionales para elevar la calidad de vida del mismo. Para hacer valer estos derechos, el estado se apoya en organismos (Ministerio del Trabajo, Organización Internacional del TrabajoOIT) donde el principal objetivo público es velar para que se cumplan las leyes (Código del Trabajo, Ley de Fomento, Reglamentos, Gacetas Judiciales), decretos y providencias en materia laboral para fortalecer los derechos del capital humano.

En este sentido, el Código del Trabajo (2012) en Ecuador, es considerado como la guía que regula las relaciones entre el capital humano y el patrono, donde se desarrollan una serie de normas legales relacionadas con las condiciones de trabajo, además se resaltan leyes especiales nacionales e internacionales con la intencionalidad de mantener actualizada la legislación laboral en el país.

En dicho Código del trabajo, se resalta en los artículos números 2 y 3 la obligatoriedad del trabajo, destacando que el mismo es "un derecho y un deber social". Todo esto en concordancia con la Constitución y las leyes. También se refiere a la libertad de trabajo y contratación, donde se resalta que el capital humano es libre para dedicar su esfuerzo a la labor lícita que a bien tenga. Expresa que "Ninguna persona podrá ser obligada a realizar trabajos gratuitos, ni remunerados que no sean impuestos por la ley, salvo los casos de urgencia extraordinaria o de necesidad de inmediato auxilio".

Por lo tanto, el derecho al trabajo y la obligatoriedad de los beneficios contemplados en las diferentes normativas establecidas, se encuentran representadas en este Código laboral, que definitivamente lo que pretende es solidificar la seguridad social del capital humano y, que se cumplan, todos sus derechos así como sus deberes; para elevar la calidad de vida y realzar la economía del país.

Finalmente, el derecho del trabajo, en comparación con otras ramas del derecho, se dirige de manera directa al capital humano organizacional, dejando ver una serie de normativas legales que regularizan su estatus social dentro del ámbito laboral (Martínez, 2016).

Es importante resaltar, que la realidad social existente en el ambiente laboral, es un elemento distorsionante que hay que tomar en consideración en el entorno del trabajo. Es por esto que el ordenamiento jurídico actúa de manera coherente en dichas realidades sociales, para no perder el norte y reivindicar los derechos laborales para el crecimiento social, familiar e individual del capital humano. 
Este ordenamiento jurídico, en el caso de Ecuador, se ajusta a las normativas laborales existentes entre las que se destacan: Leyes orgánicas, ordinarias, reglamentos, Gacetas jurídicas, etcétera, que permiten soportar de manera incondicional lo estipulado o expuesto en la Constitución ecuatoriana en cuanto a que el trabajo es un derecho y un deber social.

Todo esto, sin descuidar lo destacado en el plan nacional del buen vivir (2017), donde se, desarrollan temas de interés laboral exaltando la posibilidad de que el capital humano organizacional crezca de manera exponencial en su ambiente de trabajo. Cabe destacar, que en el objetivo 9 del plan nacional para el buen vivir, concuerda de manera explícita con lo estipulado en la Constitución de la república de Ecuador en su artículo №276, donde concuerdan que el desarrollo en el trabajo debe basarse en la generación de un empleo digno y estable.

Lo interesante e importante de todo lo descrito anteriormente, es que mediante estos organismos, el estado puede garantizar de manera consistente la generación de empleos dignos, las remuneraciones justas y acordes a las competencias del capital humano, ambientes de trabajo acordes y saludables, estabilidad laboral para el mejoramiento de una vida digna, el principio de igualdad y el derecho a que el capital humano se organice dentro de las empresas mediante sindicatos laborales que sólo persiguen el beneficio de todos sus integrantes (Briones, 2017).

Hoy se puede evidenciar el crecimiento que ha experimentado Ecuador en materia laboral y de resguardo de su capital humano dentro de las organizaciones (Flores, 2016). Parte de este crecimiento, es producto de la generación de empleos que se vienen suscitando y todas las garantías laborales que se han establecido así como, las oportunidades de empleos generadas por las diferentes inversiones a nivel nacional, que se vienen generando para lograr el sostenimiento económico que respalde al país de manera prospectiva. Todo esto, enmarcado dentro de las actividades existentes entre un estatus tripartita (Estado, Empresa y Capital Humano) entre las que podemos destacar, mejoramiento del Código Laboral, ampliación del programa Buen Vivir; donde lo más importante sea el mejoramiento de la calidad de vida del trabajador y el direccionamiento hacia los derechos laborales del mismo (INEAF, 2018).

\section{Complejidad del capital humano en las empresas desde el punto de vista legal}

El capital humano dentro de las organizaciones, pasa por situaciones tan complejas a lo largo de su estadía, que surge la necesidad de implementar procesos multidisciplinarios que permitan la convivencia empresarial (Rodríguez, 2016).

Es de destacar, que la mente humana, no existe sin la conexión del cerebro, y tampoco con la exclusión de situaciones afectivas tales como tradiciones familiares, sociales, laborales, culturales y étnicas (Rodríguez y Contreras, 2014). Lo que se quiere destacar, es que mientras más se entiende todo el sistema humano, más se reduce la experiencia a sectores limitados del conocimiento, entrando en acción el pensamiento reduccionista, como elemento integrador social.

Ante esta posición, Morín (1996) expresa que:

La necesidad del pensamiento complejo no sabrá ser justificada en algunas líneas. Tal 
necesidad no puede más que imponerse progresivamente a lo largo de un camino en el cual aparecerán, ante todo, los límites, las insuficiencias y las carencias del pensamiento simplificante, es decir, las condiciones en las cuales no podemos eludir el desafío de lo complejo. (p.22)

Por lo tanto, la complejidad del capital humano dentro de las organizaciones, se enmarcan dentro de los paradigmas emergentes, debido a su caracterización de sostener la realidad empresarial de sus procesos no ordenables o programables desde el exterior, para la obtención de escenarios diversos que permitan el logro de objetivos gerenciales para la toma de decisión (Martínez, 200).

En relación con esto último, para adaptar estos paradigmas emergentes, es bueno aclarar que los mismos van referidos a los procesos de cambios o transformación que las organizaciones necesitan, para lograr que sus procesos internos obtenga un estatus de transición y se puedan evidenciar estos cambios. Estos paradigmas emergentes, pudieran darse de dos maneras, la primera modifica por alguna razón en concreto, por convivencia o porque no se logra comprender y la segunda por su resistencia al cambio.

Significa entonces, que según el tipo o actividad de la empresa unido a la necesidad de realizar importantes cambios en materia laboral, es necesario emplear el primero de ellos, por la simple razón de que estimula la necesidad de hacer algo distinta, desarrollando procesos de innovación y creatividad que por lo general, terminan siendo elementos de estrategia que permiten crecer operativa y financieramente a las organizaciones.

Ahora bien, dentro de las propiedades que envuelven a la complejidad y, enfocándolo hacia la legalidad del capital humano en las organizaciones, Morin (2010) destaca lo conocido como la Auto-organización, de allí que para comprender su dinámica compleja organizacional, es necesario entender dos conceptos básicos. El primero de ellos es la entropía, la cual es de tendencia natural dentro de un proceso de desorden interno, y el segundo, la negentropía que contradice a la entropía, y es conocida como la presión que es empujada por alguien, para preservar el orden interno de la organización.

La idea es que como la complejidad se hace presente dentro del proceso legal organizacional del capital humano, lo que se pretende es encontrar ese punto de equilibrio para reorientar el orden y el desorden, para luego adaptar la auto-organización y la disciplina, apoyados en el respeto a las Leyes y a los derechos del capital humano en el trabajo.

Es así como, paradójicamente debemos entender que el orden de la organización viviente no es simple y depende de una lógica de complejidad. Ahora bien, dentro de los principios que expone Morin (2000) relacionados con la complejidad y, que tienen relación directa con la investigación, destaca el de la recursividad organizacional.

Dicho principio destaca que, todo lo que es producido regresa a aquello que lo produjo, convirtiéndose en productor, es decir, determina que los productos y los efectos son, simultáneamente, causas y productores de aquello que los producen. Este principio de recursividad desintegra toda idea de causa-efecto y de producto-productor, porque constituye un 
ciclo auto-constitutivo, auto-organizador y auto-productor a la vez.

Afianzando que todos los procesos legales que envuelven al capital humano dentro de las organizaciones, de alguna manera se retribuirán de forma positiva para ambos actores (trabajadorpatrono) convirtiéndose al mismo tiempo, en aumento del progreso, rentabilidad y por ende, crecimiento organizacional.

En definitiva, la complejidad del capital humano en las organizaciones no se caracteriza solamente desde el punto de vista legal. También se hace presente en la ciencia, en la sociedad, en la ética y hasta en la misma política, por lo tanto se debe tratar como un problema de pensamiento y de paradigma.

Dentro de este complejo proceso, existen dos tipos de saberes distorsionadores: quien no sabe y quiere aprender más sobre los beneficios legales dentro de la organización; y la más peligrosa, que es quien cree que el conocimiento es un proceso lineal, que avanza haciendo luz donde antes existía oscuridad, ignorando que todo conocimiento nuevo y prospectivo produce, cambios de tipo transicional, que se traduce en crecimiento, productividad, innovación y mejor calidad de vida.

\section{Materiales y Métodos.}

La importancia que reviste validar y recolectar la información en la investigación, cada vez toma relevancia dentro del mundo científico (Hernández, Méndez, Mendoza y Cuevas, 2017). El mismo tiene como fundamento principal, medir la efectividad de los resultados y la aplicación certera de los instrumentos, adaptando su utilidad para verificar epistemológicamente resultados científicos satisfactorios (Hernández-Sampieri y Mendoza, 2018).

El método interpretativo, resalta las incertidumbres del investigador hacia la realidad observada (Gómez, 2011). La investigación interpretativa recupera la subjetividad en el estudio, además de rescatar la importancia de las acciones y del comportamiento de los actores o sujetos analizados. En este método de investigación no se plantean hipótesis previas, ni marco teórico precedente. Se inicia con preguntas o cuestionamientos que permitan acercarse a la realidad que se pretende estudiar y al objeto de estudio (Behar, 2008).

La teoría acompaña a la investigación durante todo su recorrido, como apoyo teórico metodológico, como apoyo teórico conceptual y las llamadas conceptualizaciones implícitas; privilegiando el aporte teórico en el momento de la construcción de categorías analíticas, y en lo que se llama debate teórico (Baena, 2014).

Por lo tanto, concluimos que el método interpretativo es el conjunto de perspectivas teóricas mutuamente interrelacionadas y que comparten orientaciones metodológicas en la práctica de la investigación cualitativa. Al mismo tiempo, analiza los fenómenos sociales en su medio natural, para generar conocimiento científico, mediante la comprensión e interpretación hermenéutica de los textos (Domínguez, 2015).

Entre los elementos que se conjugan para la obtención de resultados satisfactorios, Rodríguez 
(2018) expone que en las conclusiones investigativas relacionadas con el método interpretativo, destacan: (a) la compresión de ideas y realidades dentro de un contexto; (b) el estudio holístico como referencia a los puntos de vistas múltiples que interaccionan en el medio; (c) el surgimiento de la reflexión acercándose de manera clara y precisa a la práctica; (d) la importancia hacia la comprensión más que a la confirmación; (e) descripciones enriquecedoras enmarcadas dentro de la hermenéutica y, finalmente, (f) donde se profundizan los estudios y se pone en práctica la metodología cualitativa.

\section{Discusiones y Conclusiones}

El capital humano representa el motor principal y el activo más importante y relevante en las organizaciones. El adecuado manejo del mismo, trae como consecuencia resultados favorables que sirven para el crecimiento organizacional en todos los ámbitos de la empresa.

Toda organización necesita reorientar sus operaciones en concordancia y apoyo con su capital humano, tendrá altas probabilidades de poder cubrir o alcanzar todos los objetivos gerenciales que se planteen. Mediante esta investigación, queda evidenciada la importancia que reviste cumplir con todos los beneficios contractuales desde el punto de vista laboral, con el capital humano, ya que formaría parte de las políticas Constitucionales y sociales para el crecimiento y el buen vivir del trabajador.

Desde el punto de vista legal laboral, es importante que los dueños de las organizaciones cumplan con todo lo estipulado en las Leyes, ya que de esta manera su compromiso social se recompensará con la calidad del trabajo que le prestan sus empleados. Es importante resaltar, que pueden existir situaciones distorsionantes que de alguna manera afecte de manera directa las directrices gerenciales, por lo tanto, entre las recomendaciones que resultan de esta investigación podemos nombrar: que gerenciar la crisis con una planificación estratégica apropiada para entornos volátiles, será de gran ayuda para solventar los problemas internos y externos de la organización.

También la gestión del capital humano debe estar alineada con la planificación general de la compañía. La constante revisión de los contratos laborales para mejorarlos o mantenerlos. Revisiones constantes de forma periódica sobre las compensaciones y beneficios del capital humano. Revisión constante de entes económicos tales como la inflación para ajustar los ingresos del trabajador. Llevar adelante planes de formación, para acortar las brechas entre las competencias requeridas y las disponibles en el mercado.

En este mismo orden de ideas, definir planes de carrera y de crecimiento profesional, especialmente para el personal clave, a fin de que se pueda manejar mejor la incertidumbre. Identificar elementos de motivación para retener los talentos, fundamentalmente en entornos de pesimismo. Mejorar las comunicaciones y hacerlas más transparentes entre todos los integrantes de la organización. Asegurar que el clima de trabajo sea adecuado, con confianza entre las partes, y que todos los miembros de la organización estén alineados hacia una misma meta de producción. 
Finalmente, implantar planes que incentiven la innovación para lograr mayor eficiencia, productividad y dinamismo, de forma tal que la empresa se torne competitiva dentro del mercado donde se desenvuelve.

\section{Referencias bibliográficas}

Alfonso, R. (2019). La importancia del capital humano en la organización. (Monografía Post-grado). Fundación Universidad de América. Bogotá, Colombia.

Baena, G. (2014). Metodología de la investigación. Serie integral por competencia. México: Grupo Editorial Patria.

Barroso, M. (2011). Meditaciones gerenciales. Caracas, Venezuela: Galac, S.A.

Bazán, J. (1997). Fuentes del derecho del trabajo. México. Recuperado de https://archivos.juridicas.unam.mx/ www/bjv/libros/1/139/6.pdf (05/12/2018).

Behar, D. (2008). Metodología de la investigación. México: Shalom.

Blanco, E. (2017). Cómo sobrevive una empresa en un entorno crónicamente incierto. Debates IESA, XXI(4), 5-10.

Buen, N. (2015). Los contratos colectivos de trabajo de protección, Latinoamericana de Derecho Social, 20(1), 35-42.

Briones, M. (11 de febrero de 2017). La evolución histórica del derecho laboral en Ecuador. Presentación recuperada de https://prezi.com/loyvoaw0rngg/laevolucion-historica-derecho-laboral-enecuador.

Código del trabajo. (2012). Codificación 17. Comisión de legislación y codificación.

Constitución de la República del Ecuador. (2008). Capítulo segundo. Derechos del buen vivir. Sección octava. Trabajo y seguridad social, Artículo 33.

Domínguez, J. (2015). Manual de metodología de la investigación científica. Chimbote, Perú: ULADECH.

Dulzaides, M. y Molina, A. (2004). Análisis documental y de información: Dos componentes de un mismo proceso. EBSCO, 12(2), 63-78.

Drucker, P. (2000). El Ejecutivo Eficaz. Buenos Aires, Argentina: Sudamericana S.A

Etkin, J. (2005). Gestión de la complejidad en las organizaciones. Buenos Aires, Argentina: Ediciones Granica C.A. 
Etkin, J. y Schvarstein, L. (2000). Identidad de las organizaciones - Invariancia y cambio. Buenos Aires, Argentina: Paidós.

Firth, D. (2000). Smart. Santa Fe de Bogotá, Colombia: McGraw-Hill Interamericana.

Flores, J. (2016). Problemáticas laborales en el Ecuador. Derecho Ecuador.com. Recuperado de https://www. derecho ecuador.com/problemática-laboral-en-el-ecuador.

Francés, A. (2008). Antología. Debates IESA: Competitividad, gerencia y estrategias. Homenaje a Antonio Francés. Caracas, Venezuela: Instituto de Estudios Superiores de Administración (IESA).

García, J. (2015). Compendio del derecho del trabajo. España: Tiedra.

Godin, S. (2015). Poke the box. Nueva York: Brilliance Corp.

Gómez, E. (9 de octubre de 2011). El modelo interpretativo tercer semestre. [Mensaje en un blog]. Recuperado de http://ejeme tupnle94.blogspot.com/2011/10/el-model o-interpretativo-tercer.html

Hernández-Sampieri, R. y Mendoza, C. (2018). Metodología de la investigación. Las rutas cuantitativa, cualitativa y mixta. México: McGrawHill Education.

Hernández, R., Méndez, S., Mendoza, C. y Cuevas, A. (2017). Fundamentos de investigación. México: McGrawHill Education.

INEAF (05 de diciembre de 2018). Fuentes del derecho laboral. Recuperado de https://www.ineaf.es/divulgativo/ laboral/fuentes-del-derecho-laboral.

Kin, J. (2017). Countries commit to strong action on human capital to drive economic growth. The World Bank. Recuperado de http://www.worldbank.org/en/news/feature/2017/10/20/countries-commit-to-strong -actionon-human-capital-to-drive-econo mic-growth.

López, S. (2015). Justicia Cotidiana, Síntesis del informe y de las recomendaciones en materia de justicia cotidiana. México: Centro de investigación y docencia. Económica, A.C.

López, M. y Grandío, A. (2005). Capital humano como fuente de ventajas competitivas. España: Gesbiblo Ediciones.

Martínez M. (2008). Ciencia y arte en la metodología cualitativa. México: Trillas.

Martínez, V. (2016). Reforma al derecho procesal del trabajo. El exterminio de las juntas de conciliación y arbitraje. Letras jurídicas, 35(1), 125-142.

Morín, E. (2000). Introducción al pensamiento complejo. Barcelona, España: Gedisa. 
(1996). Mis demonios. Barcelona, España: Kairós.

Palomo, I. (2008). Fundamentos del derecho laboral. Lus et Praxis 14(2), 659-665.

Penfold, M. y Vainrub, R. (2012). Estrategias en tiempos de turbulencia: Las empresas venezolanas. Caracas, Venezuela: IESA.

Plan Nacional del Buen Vivir. (2017). Toda una vida. Secretaria nacional de planificación y desarrollo [Semplades]. Quito, Ecuador

Rodríguez, A. (17 de marzo de 2018). Paradigma interpretativo en investigación: Características y autores. [Mensaje en un blog]. Recuperado de https://www.lifeder. coparadigma-interpretativo-investigacion.

Rodríguez, L. (2016). La emergencia de los enfoques de la complejidad en América Latina. Buenos Aires, Argentina: La Imprenta Digital SRL.

Rodríguez, A. y Contreras, O. (2014). El quehacer de las ciencias sociales: Una visión desde adentro. Tijuana, México: COMECSO.

Sull, D., Homkes, R. y Sull, C. (2015). Why strategy execution unravels and what to do about it. Harvard Business Review, 93(3), 15-27.

Torres, C. (2018). Formación y desarrollo: Éxito y tendencias. Debates IESA, XXIII(1), 14-19.

Tushman, M., Smith, W. y Binns, A. (2011). The Ambidextrous CEO. Harvard Business Review, 89(6), 115-126.

Vargas, F. (2002). Competencias en la formación y competencias en la gestión del talento humano convergencias y desafíos. Consultor CINTERFOR/OIT. Publicaciones del Banco Interamericano de Desarrollo. 